\title{
A NOTE ON LONG-TERM OPTIMAL PORTFOLIOS UNDER DRAWDOWN CONSTRAINTS
}

\author{
JUN SEKINE, ${ }^{*}$ Kyoto University
}

\begin{abstract}
The maximization of the long-term growth rate of expected utility is considered under drawdown constraints. In a general situation, the value and the optimal strategy of the problem are related to those of another 'standard' risk-sensitive-type portfolio optimization problem. Furthermore, an upside-chance maximization problem of a large deviation probability is stated as a 'dual' optimization problem. As an example, a 'linearquadratic' model is studied in detail: the conditions to ensure the solvabilities of the problems are discussed and explicit expressions for the solutions are presented.
\end{abstract}

Keywords: Drawdown constraint; risk constraint; long-term investment; risk-sensitive control; Skorokhod equation; algebraic Riccati equation

2000 Mathematics Subject Classification: Primary 93E20; 91B28; 90C39; 49N10

\section{Introduction}

Consider a financial market consisting of a bank account and $n$ risky assets. Let $S^{0}$ be the

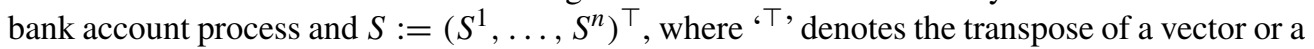
matrix, the price process of the $n$ risky assets, both of which are modelled on $\left(\Omega, \mathcal{F}, \mathrm{P},\left(\mathcal{F}_{t}\right)_{t \geq 0}\right)$, a complete probability space endowed with a filtration satisfying the usual conditions. Assume that $S^{0}$ is an adapted, continuous, nondecreasing process starting at $S_{0}^{0}=1$, and that $S$ is a continuous semimartingale such that $S^{i}>0$ for all $i, 1 \leq i \leq n$. Now consider an investor whose wealth process is governed by the equation

$$
\begin{aligned}
\mathrm{d} X_{t}^{\alpha, \pi}= & \sum_{i=1}^{n}\left(X_{t}^{\alpha, \pi}-\alpha S_{t}^{0} \tilde{M}_{t}^{\alpha, \pi}\right) \pi_{t}^{i} \frac{\mathrm{d} S_{t}^{i}}{S_{t}^{i}} \\
& +\left\{\left(1-\sum_{i=1}^{n} \pi_{t}^{i}\right)\left(X_{t}^{\alpha, \pi}-\alpha S_{t}^{0} \tilde{M}_{t}^{\alpha, \pi}\right)+\alpha S_{t}^{0} \tilde{M}_{t}^{\alpha, \pi}\right\} \frac{\mathrm{d} S_{t}^{0}}{S_{t}^{0}}, \\
X_{0}^{\alpha, \pi}= & x,
\end{aligned}
$$

with $x>0, \alpha \in[0,1)$, and $\tilde{M}_{t}^{\alpha, \pi}:=\max _{s \in[0, t]} X_{s}^{\alpha, \pi} / S_{s}^{0}$, which was introduced and studied in the interesting papers [8] and [5]. Here $\pi:=\left(\pi^{1}, \ldots, \pi^{n}\right)^{\top}$ is a progressively measurable process such that

$$
\sum_{i, j=1}^{n} \int_{0}^{T} \pi_{t}^{i} \pi_{t}^{j} \frac{\mathrm{d}\left\langle S^{i}, S^{j}\right\rangle_{t}}{S_{t}^{i} S_{t}^{j}}<\infty \quad \text { and } \sum_{i=1}^{n} \int_{0}^{T}\left|\pi_{t}^{i}\right| \frac{\mathrm{d} S_{t}^{0}}{S_{t}^{0}}<\infty
$$

Received 10 February 2006.

* Postal address: Institute of Economic Research, Kyoto University, Yoshida-Honmachi, Sakyo-ku, Kyoto, 606-8501, Japan. Email address: sekine@kier.kyoto-u.ac.jp 
and represents the trading strategy of the investor as explained below. We denote by $\langle\cdot, \cdot\rangle$ the quadratic covariance process. Recalling that, by the product rule,

$$
\tilde{X}_{t}^{\alpha, \pi}:=\frac{X_{t}^{\alpha, \pi}}{S_{t}^{0}} \quad \text { and } \quad \tilde{S}_{t}^{i}:=\frac{S_{t}^{i}}{S_{t}^{0}}
$$

respectively satisfy

$$
\mathrm{d} \tilde{X}_{t}^{\alpha, \pi}=\frac{\mathrm{d} X_{t}^{\alpha, \pi}}{S_{t}^{0}}-\frac{X_{t}^{\alpha, \pi} \mathrm{d} S_{t}^{0}}{\left(S_{t}^{0}\right)^{2}} \quad \text { and } \quad \frac{\mathrm{d} \tilde{S}_{t}^{i}}{\tilde{S}_{t}^{i}}=\frac{\mathrm{d} S_{t}^{i}}{S_{t}^{i}}-\frac{\mathrm{d} S_{t}^{0}}{S_{t}^{0}},
$$

we rewrite (1.1) as

$$
\begin{aligned}
\mathrm{d} \tilde{X}_{t}^{\alpha, \pi} & =\left(\tilde{X}_{t}^{\alpha, \pi}-\alpha \tilde{M}_{t}^{\alpha, \pi}\right) \sum_{i=1}^{n} \pi_{t}^{i} \frac{\mathrm{d} \tilde{S}_{t}^{i}}{\tilde{S}_{t}^{i}}, \\
\tilde{X}_{0}^{\alpha, \pi} & =x .
\end{aligned}
$$

Its unique (adapted) solution is given by

$$
\tilde{X}_{t}^{\alpha, \pi}=x\left[(1-\alpha) \exp \left\{L_{t}^{\pi}-\alpha \max _{s \in[0, t]} L_{s}^{\pi}\right\}+\alpha \exp \left\{(1-\alpha) \max _{s \in[0, t]} L_{s}^{\pi}\right\}\right],
$$

where

$$
L_{t}^{\pi}:=\sum_{i=1}^{n} \int_{0}^{t} \pi_{s}^{i} \frac{\mathrm{d} \tilde{S}_{s}^{i}}{\tilde{S}_{s}^{i}}-\frac{1}{2} \sum_{i, j=1}^{n} \int_{0}^{t} \pi_{s}^{i} \pi_{s}^{j} \frac{\mathrm{d}\left\langle\tilde{S}^{i}, \tilde{S}^{j}\right\rangle_{s}}{\tilde{S}_{S}^{i} \tilde{S}_{s}^{j}},
$$

which satisfies the relation

$$
\tilde{M}_{t}^{\alpha, \pi}=x \exp \left\{(1-\alpha) \max _{s \in[0, t]} L_{s}^{\pi}\right\} .
$$

These facts were observed in the appendix of [5], using a unique solution to the Skorokhod equation associated with the continuous process $L^{\pi}$ (see Lemma 3.6.14 of [13], for example). We thus see, from (1.3) and (1.5), that

$$
X_{t}^{\alpha, \pi}-\alpha S_{t}^{0} \tilde{M}_{t}^{\alpha, \pi}=(1-\alpha) x S_{t}^{0} \exp \left\{L_{t}^{\pi}-\alpha \max _{s \in[0, t]} L_{s}^{\pi}\right\}>0
$$

in other words, the drawdown constraint

$$
\mathrm{P}\left(X_{t}^{\alpha, \pi}>\alpha S_{t}^{0} \tilde{M}_{t}^{\alpha, \pi} \text { for all } t \in[0, \infty)\right)=1
$$

is always satisfied. Equation (1.1) now states that the investor

- starts with the initial capital $x>0$

and, at time $t$,

- invests a proportion $\pi_{t}^{i}$ of the difference $X_{t}^{\alpha, \pi}-\alpha S_{t}^{0} \tilde{M}_{t}^{\alpha, \pi}$ in the $i$ th risky asset and

- keeps the remainder, $\left(1-\sum_{i=1}^{n} \pi_{t}^{i}\right)\left(X_{t}^{\alpha, \pi}-\alpha S_{t}^{0} \tilde{M}_{t}^{\alpha, \pi}\right)+\alpha S_{t}^{0} \tilde{M}_{t}^{\alpha, \pi}$, in the bank. 
Here recall that the strategy is self-financing, i.e.

$$
\sum_{i=1}^{n} \pi_{t}^{i}\left(X_{t}^{\alpha, \pi}-\alpha S_{t}^{0} \tilde{M}_{t}^{\alpha, \pi}\right)+\left\{\left(1-\sum_{i=1}^{n} \pi_{t}^{i}\right)\left(X_{t}^{\alpha, \pi}-\alpha S_{t}^{0} \tilde{M}_{t}^{\alpha, \pi}\right)+\alpha S_{t}^{0} \tilde{M}_{t}^{\alpha, \pi}\right\}=X_{t}^{\alpha, \pi}
$$

and that it allows short-sellings, i.e. the proportion $\pi_{t}^{i} \in \mathbb{R}$ can take negative values.

For an investor having the wealth process (1.1) with the drawdown constraint (1.6), the authors of [8] and [5] treated the following maximization problem for the long-term growth rate of the expected utility of wealth:

$$
\Gamma_{\alpha}(\gamma):=\sup _{\pi \in \mathcal{A}} \varlimsup_{T \rightarrow \infty} \frac{1}{T} \log \mathrm{E}\left(X_{T}^{\alpha, \pi}\right)^{\gamma} .
$$

Here $\gamma \in(0,1 /(1-\alpha))$ and $\mathcal{A}$ is the totality of progressively measurable processes whose element $\pi:=\left(\pi_{t}\right)_{t \geq 0}$ satisfies (1.2). In particular, using special models, they gave explicit solutions to the problems considered. The authors of [8] treated the Black-Scholes economy in which the risk-free interest rate of the bank account is a constant and the single risky asset price process is given by a one-dimensional lognormal diffusion with one-dimensional Brownian motion and constant coefficients. Furthermore, the authors of [5] treated a generalized situation in which the risk-free interest rate is deterministic and the multidimensional risky asset price processes are defined by an $n$-dimensional lognormal stochastic differential equation with $n$ dimensional Brownian motion and deterministic coefficients.

In this paper, we aim to extend their results for more general models of the processes $S^{0}$ and $S$. Indeed, under the setting we have prepared, and introducing another optimization problem,

$$
\Lambda_{\alpha}(\gamma):=\sup _{\pi \in \mathcal{A}} \varlimsup_{T \rightarrow \infty} \frac{1}{T} \log \mathrm{E}\left(S_{T}^{0} \mathrm{e}^{(1-\alpha) L_{T}^{\pi}}\right)^{\gamma}
$$

we obtain the following theorem.

Theorem 1.1. Let $\alpha \in[0,1)$ and $\gamma \in(0,1 /(1-\alpha))$ be given. Assume that $\Lambda_{(\cdot)}(\gamma)$ is upper semicontinuous on the left at $\alpha$ if $\alpha>0$ and $\Lambda_{\alpha}(\gamma)<\infty$. Then

$$
\Gamma_{\alpha}(\gamma)=\Lambda_{\alpha}(\gamma)
$$

Furthermore, a strategy is optimal for the problem (1.7) if it is optimal for the problem (1.8), and a strategy $\hat{\pi} \in \mathcal{A}$ attains the maximum for (1.7) with a limit

$$
\Gamma_{\alpha}(\gamma)=\lim _{T \rightarrow \infty} \frac{1}{T} \log \mathrm{E}\left(X_{T}^{\alpha, \hat{\pi}}\right)^{\gamma}
$$

if it attains the maximum for (1.8) with a limit

$$
\Lambda_{\alpha}(\gamma)=\lim _{T \rightarrow \infty} \frac{1}{T} \log \mathrm{E}\left(S_{T}^{0} \mathrm{e}^{(1-\alpha) L_{T}^{\hat{\hat{n}}}}\right)^{\gamma}
$$

Remark 1.1. Let $\alpha \in[0,1)$ be given. We can consider another problem,

$$
\Pi_{\alpha}(k):=\sup _{\pi \in \mathcal{A}} \varlimsup_{T \rightarrow \infty} \frac{1}{T} \log \mathrm{P}\left(X_{T}^{\alpha, \pi} \geq \mathrm{e}^{k T}\right),
$$


i.e. the maximization problem of the large deviation probability of beating the target growth rate $k \in \mathbb{R}$ in the long run. For $\alpha=0$, such a problem was introduced in [17] and [18] and studied via the 'dual' optimization problem (1.7) or (1.8). For nonzero $\alpha$, we can assert the following 'dual' characterization of the large deviation control problem (1.12), tracing the proof of Theorem 3.1 of [18].

Suppose, for any $\gamma \in(0, \bar{\gamma}), \bar{\gamma}>0$, that there exists an optimal strategy

$$
\hat{\pi}_{\alpha}(\gamma):=\left(\hat{\pi}_{\alpha}(t, \gamma)\right)_{t \geq 0} \in \mathcal{A}
$$

for (1.7), establishing (1.10) with $\hat{\pi}:=\hat{\pi}_{\alpha}(\gamma)$. Suppose also that $\Gamma_{\alpha} \in C^{1}((0, \bar{\gamma}))$, where $C^{1}(A)$ denotes the space of continuously differentiable functions with (generic) domain $A$. Define $I_{\alpha}:\left(\Gamma_{\alpha}^{\prime}(0+), \Gamma_{\alpha}^{\prime}(\bar{\gamma}-)\right) \rightarrow(0, \bar{\gamma})$ to be the inverse of $\left.\Gamma_{\alpha}^{\prime}(\cdot)\right|_{(0, \bar{\gamma})}$, i.e. $I_{\alpha}\left(\Gamma_{\alpha}^{\prime}(d)\right)=d$. Here $\Gamma_{\alpha}^{\prime}(\gamma):=(\mathrm{d} / \mathrm{d} \gamma) \Gamma_{\alpha}(\gamma)$. Then

$$
\Pi_{\alpha}(k)=-\sup _{\gamma \in(0, \bar{\gamma})}\left\{\gamma k-\Gamma_{\alpha}(\gamma)\right\}
$$

for all $k<\Gamma_{\alpha}^{\prime}(\bar{\gamma}-)$, and the sequence of controls $\left(\hat{\pi}^{[k, n]}\right)_{n \in \mathbb{N}}$ defined by

$$
\hat{\pi}_{t}^{[k, n]}:=\hat{\pi}_{\alpha}\left(t, I_{\alpha}\left(\left(k \vee \Gamma_{\alpha}^{\prime}(0+)\right)+1 / n\right)\right)
$$

is nearly optimal, i.e.

$$
\lim _{n \rightarrow \infty} \lim _{T \rightarrow \infty} \frac{1}{T} \log \mathrm{P}\left(X_{T}^{\alpha, \hat{\pi}^{[k, n]}} \geq \mathrm{e}^{k T}\right)=\Pi_{\alpha}(k) \quad \text { for } k<\Gamma_{\alpha}^{\prime}(\bar{\gamma}-) .
$$

Here note that extra effort is required to solve (1.12): a regularity of the 'dual' value function $\Gamma_{\alpha}(\cdot)$ is necessary along with its solvability on a certain interval $(0, \bar{\gamma})$, and, actually, to determine the maximal interval in the effective domain, $\mathscr{D}_{\alpha}:=\left\{\gamma>0: \Gamma_{\alpha}(\gamma)<\infty\right\}$, is a rather complicated problem (see Remark 2.3 and Theorem 2.4 and the statements immediately before it).

Remark 1.2. Remark 1.1 states that the investor can determine the risk-averse parameter $I_{\alpha}(k \vee$ $\left.\Gamma_{\alpha}^{\prime}(0+)\right)$ in (1.7) by choosing the target growth rate $k$ in (1.12) to maximize the upside-chance probability of beating the target growth rate $k$ in an asymptotic way.

On the other hand, the investor can control the downside-risk of the portfolio wealth process (1.1) in (1.7) or (1.12) by choosing $\alpha \in[0,1)$ suitably. Suppose, for example, that at time 0 the investor monitors the future losses, $\left(-\left(X_{t}^{\alpha, \pi}-x S_{t}^{0}\right)\right)_{t \geq 0}$, of the portfolio wealth process using the risk measure

$$
\begin{aligned}
\rho_{0}\left(-\left(X^{\alpha, \pi}-x S^{0}\right)\right): & =\sup \left\{\mathrm{E}^{\mathrm{Q}}\left(\left(S_{\tau}^{0}\right)^{-1}\left\{-\left(X_{\tau}^{\alpha, \pi}-x S_{\tau}^{0}\right)\right\}\right): \mathrm{Q} \in \mathcal{P}\right\} \\
& =x-\inf \left\{\mathrm{E}^{\mathrm{Q}}\left(\tilde{X}_{\tau}^{\alpha, \pi}\right): \mathrm{Q} \in \mathcal{P}\right\},
\end{aligned}
$$

where $\mathcal{P}$ is a given set of probability measures on $(\Omega, \mathcal{F}), \mathrm{E}^{\mathrm{Q}}$ denotes expectation with respect to $\mathrm{Q}$, and $\tau$ denotes the stopping time. Risk measures such as $\rho_{0}(\cdot)$ for processes possess important 'coherent' properties; see [1] or [4], for example. (Here we comment only on the risk measurement at time 0 . Conditions should be imposed on the set $\mathcal{P}$ when we consider a time-consistent monitoring of the risk; see [1] and [4].) From the above expression, we see that

$$
\rho_{0}\left(-\left(X^{\alpha, \pi}-x S^{0}\right)\right) \leq(1-\alpha) x,
$$

where we use (1.6) and the fact that $\tilde{M}^{\alpha, \pi}$ is nondecreasing. This implies that the investor can control the upper bound of (the risk of) the future losses by selecting $\alpha \in[0,1)$. 
As a consequence of Theorem 1.1, in solving (1.7) it is crucial to investigate the solvability of (1.8). Furthermore, in solving (1.12) we need to check the regularity of the value function $\Lambda_{(\cdot)}(\cdot)$. The problem $(1.8)$, or its variation

$$
\inf _{\pi} \lim _{T \rightarrow \infty} \frac{1}{T} \log \mathrm{E}\left(S_{T}^{0} \mathrm{e}^{(1-\alpha) L_{T}^{\pi}}\right)^{\gamma}, \quad \gamma<0
$$

is included in risk-sensitive-type portfolio optimization problems, which have been studied by several authors by analysing the associated Bellman equations with Markovian models; see [2], [12], [16], and the references therein, for example. We note that there are different difficulties in the analyses of the respective situations in which $\gamma<0$ and $\gamma>0$. For the former case, conditions to ensure the solvabilities of the Bellman equations and the optimization problems were provided in [12] and [16] in a fairly general Markovian setting. However, these conditions are not applicable in the latter case, and to the best of the author's knowledge no such general results have been obtained in that case.

In the next section, we consider an example of a model in which $S^{0}$ has stochastic interest rates and $S$ has stochastic mean return rates which we assume to have 'linear-quadratic' structures in order to discuss the solvabilities. In such a situation, this reduces to discussing the solvability of the related algebraic Riccati equation. It is sufficient to show the existence of its stabilizing solution (see Theorem 2.1), and this existence is always ensured on a certain interval, $J \times(0, \bar{\gamma})$, in the parameter space $\{(\alpha, \gamma) \in[0,1) \times(0,1 /(1-\alpha))\}$, under certain assumptions (see (2.1)-(2.3), Theorem 2.3, and Corollary 2.1). Moreover, we can give an 'almost' necessary and sufficient condition for the solvability of (1.8) if the 'stochastic factor' is one-dimensional (see Theorem 2.4).

All the proofs of the results of Section 1 and Section 2 are collected in Section 3, and in Section 4 we conclude.

\section{A 'linear-quadratic' example}

In this section, we work in the following setting. Let $\left(\Omega, \mathcal{F}, \mathrm{P},\left(\mathcal{F}_{t}\right)_{t \geq 0}\right)$ be a filtered probability space with an augmented Brownian filtration generated by the $(m+n)$-dimensional Brownian motion $w:=\left(w_{1}, \ldots, w_{m+n}\right)^{\top}$, and suppose that $S^{0}$ and $S$ are described by

$$
\begin{aligned}
\mathrm{d} S_{t}^{0} & =S_{t}^{0} r\left(Y_{t}\right) \mathrm{d} t, & & S_{0}^{0}=1, \\
\mathrm{~d} S_{t} & =\operatorname{diag}\left(S_{t}\right)\left\{\mu\left(Y_{t}\right) \mathrm{d} t+\sigma\left(Y_{t}\right) \mathrm{d} w(t)\right\}, & & S_{0} \in \mathbb{R}_{+}^{n}, \\
\mathrm{~d} Y_{t} & =\lambda\left(Y_{t}\right) \mathrm{d} t+\eta\left(Y_{t}\right) \mathrm{d} w(t), & & Y_{0} \in \mathbb{R}^{m},
\end{aligned}
$$

using the $m$-dimensional stochastic factor process $Y$, which affects the mean return rate, $\mu\left(Y_{t}\right)$, of $S$, the volatility, $\sigma\left(Y_{t}\right)$, of $S$, and the risk-free interest rate, $r\left(Y_{t}\right)$, of $S^{0}$. Here $\operatorname{diag}(x)$ denotes the diagonal $n \times n$ matrix whose $(i, i)$ th element is component $x^{i}$ of $x:=\left(x^{1}, \ldots, x^{n}\right)^{\top}$. In particular, we assume that

$$
\begin{aligned}
r(y) & :=r_{0}+r_{1}^{\top} y+\frac{1}{2} y^{\top} r_{2} y, \\
\mu(y) & :=r(y) \mathbf{1}+\theta(y), \quad \theta(y):=\theta_{0}+\theta_{1} y, \\
\lambda(y) & :=\lambda_{0}+\lambda_{1} y, \\
\eta(y) & \equiv \eta_{0}, \\
\sigma(y) & \equiv \sigma_{0},
\end{aligned}
$$


where $1:=(1, \ldots, 1)^{\top} \in \mathbb{R}^{n}, r_{0} \in \mathbb{R}, r_{1}, \lambda_{0} \in \mathbb{R}^{m}, \theta_{0} \in \mathbb{R}^{n}, r_{2}, \lambda_{1} \in \mathbb{R}^{m \times m}, \theta_{1} \in \mathbb{R}^{n \times m}$, $\eta_{0} \in \mathbb{R}^{m \times(m+n)}$, and $\sigma_{0} \in \mathbb{R}^{n \times(m+n)}$. Thus,

$r_{2}$ is symmetric and nonnegative definite,

$\lambda_{1}$ is stable, and $\sigma_{0} \sigma_{0}^{\top}$ and $\eta_{0} \eta_{0}^{\top}$ are positive definite.

Here a matrix is called stable if all of its eigenvalues lie in the open left-hand half-plane.

With a linear Gaussian stochastic factor process and a quadratic Gaussian interest rate process in this model, we study the solvability of problem (1.8) using a dynamic programming approach. Start with the finite time horizon problem

$$
\bar{V}_{0}:=\sup _{\pi \in \mathcal{A}_{T}} \log \mathrm{E}\left(S_{T}^{0} \mathrm{e}^{(1-\alpha) L_{T}^{\pi}}\right)^{\gamma},
$$

for a given $T>0$. Here $\mathcal{A}_{T}$ is a space of admissible trading strategies prescribed below. For $\pi \in \mathcal{A}_{T}$, recall that

$$
S_{t}^{0} \mathrm{e}^{(1-\alpha) L_{t}^{\pi}}=\left[\mathcal{E}\left(\int \pi^{\top} \sigma_{0} \mathrm{~d} w\right)_{t} \exp \left\{\int_{0}^{t}\left(\frac{r\left(Y_{u}\right)}{1-\alpha}+\pi_{u}^{\top} \theta\left(Y_{u}\right)\right) \mathrm{d} u\right\}\right]^{1-\alpha},
$$

where we use the notation $\mathcal{E}(Z):=\left(\mathcal{E}(Z)_{t}\right)_{t \geq 0}, \mathcal{E}(Z)_{t}:=\mathrm{e}^{Z_{t}-(1 / 2)\langle Z\rangle_{t}}$, for the stochastic exponential of a continuous semimartingale $Z$. We see that

$$
\left(S_{T}^{0} \mathrm{e}^{\left.(1-\alpha) L_{T}^{\pi}\right)^{\gamma}}=\mathcal{E}\left(\delta \int \pi^{\top} \sigma_{0} \mathrm{~d} w\right)_{T} \exp \left\{\delta \int_{0}^{T} \ell\left(Y_{t}, \pi_{t}\right) \mathrm{d} t\right\},\right.
$$

where

$$
\begin{aligned}
\delta \equiv \delta(\alpha, \gamma) & :=(1-\alpha) \gamma, \\
\ell(y, p) & :=\frac{1}{1-\alpha} r(y)+\theta(y)^{\top} p-\frac{1-\delta}{2}\left|\sigma_{0}^{\top} p\right|^{2} .
\end{aligned}
$$

So, with

$$
\begin{aligned}
& \mathcal{A}_{T}:=\left\{\pi=\left(\pi_{t}\right)_{t \in[0, T]}: \pi \text { is } n\right. \text {-dimensional and progressively measurable, } \\
& \left.\qquad \int_{0}^{T}\left|\pi_{t}\right|^{2} \mathrm{~d} t<\infty \text {, and } \mathrm{E} \mathscr{E}\left(\delta \int \pi^{\top} \sigma_{0} \mathrm{~d} w\right)_{T}=1\right\},
\end{aligned}
$$

$\partial_{y}(\cdot):=\left(\partial_{y_{1}}(\cdot), \ldots, \partial_{y_{n}}(\cdot)\right)^{\top}$, and $\partial_{y y}(\cdot):=\left(\partial_{y_{i} y_{j}}(\cdot)\right)_{1 \leq i \leq m, 1 \leq j \leq m}$, we deduce the Bellman dynamic programming equation

$$
\begin{aligned}
-\partial_{t} v & =\frac{1}{2}\left\{\operatorname{Tr}\left(\eta_{0} \eta_{0}^{\top} \partial_{y y} v\right)+\left|\eta_{0}^{\top} \partial_{y} v\right|^{2}\right\}+\sup _{\pi \in \mathbb{R}^{n}}\left\{\left(\lambda(y)+\delta \eta_{0} \sigma_{0}^{\top} \pi\right)^{\top} \partial_{y} v+\delta \ell(y, \pi)\right\}, \\
v(T, y) & =0
\end{aligned}
$$

for the value process

$$
\bar{V}_{t}:=\underset{\pi \in \mathcal{A}_{t, T}}{\operatorname{ess} \sup } \log \mathrm{E}^{(\delta \pi)}\left(\exp \left\{\delta \int_{t}^{T} \ell\left(Y_{u}, \pi_{u}\right) \mathrm{d} u\right\} \mid \mathcal{F}_{t}\right) .
$$


Here $\mathcal{A}_{t, T}$ is the restriction of $\mathcal{A}$ to the time interval $[t, T]$ and $\mathrm{E}^{(\delta \pi)}$ denotes the expectation with respect to the probability measure $\mathrm{P}^{(\delta \pi)}$ on $\left(\Omega, \mathcal{F}_{T}\right)$ defined by

$$
\left.\frac{\mathrm{dP}^{(\delta \pi)}}{\mathrm{dP}}\right|_{\mathcal{F}_{t}}:=\mathcal{E}\left(\delta \int \pi^{\top} \sigma_{0} \mathrm{~d} w\right)_{t} .
$$

The maximizer, $\check{\pi}(t, y)$, in (2.7) is given by

$$
\check{\pi}(t, y):=\frac{1}{1-\delta}\left(\sigma_{0} \sigma_{0}^{\top}\right)^{-1}\left\{\theta(y)+\sigma_{0} \eta_{0}^{\top} \partial_{y} v\right\},
$$

so we can rewrite $(2.7)$ as

$$
\begin{aligned}
-\partial_{t} v= & \frac{1}{2}\left\{\operatorname{Tr}\left(\eta_{0} \eta_{0}^{\top} \partial_{y y} v\right)+\left|\eta_{0}^{\top} \partial_{y} v\right|^{2}\right\}+\lambda(y)^{\top} \partial_{y} v \\
& +\frac{\delta}{2(1-\delta)}\left|\sigma_{0}^{\top}\left(\sigma_{0} \sigma_{0}^{\top}\right)^{-1}\left\{\theta(y)+\sigma_{0} \eta_{0}^{\top} \partial_{y} v\right\}\right|^{2}+\gamma r(y), \\
v(T, y)= & 0
\end{aligned}
$$

that is,

$$
\begin{aligned}
-\partial_{t} v & =\frac{1}{2} \operatorname{Tr}\left(\eta_{0} \eta_{0}^{\top} \partial_{y y} v\right)+\frac{1}{2}\left(\partial_{y} v\right)^{\top} \eta_{0} \hat{R}^{-1} \eta_{0}^{\top} \partial_{y} v+a(y)^{\top} \partial_{y} v+b(y), \\
v(T, y) & =0
\end{aligned}
$$

where we define functions

$$
\begin{aligned}
& a(y):=\lambda(y)+\frac{\delta}{1-\delta} \eta_{0} \sigma_{0}^{\top}\left(\sigma_{0} \sigma_{0}^{\top}\right)^{-1} \theta(y)=\hat{d}+\hat{A} y, \\
& b(y):=\gamma r(y)+\frac{\delta}{2(1-\delta)} \theta(y)^{\top}\left(\sigma_{0} \sigma_{0}^{\top}\right)^{-1} \theta(y)=\hat{f}+\hat{e}^{\top} y+\frac{1}{2} y^{\top} \hat{C} y,
\end{aligned}
$$

and matrices, vectors, and a constant

$$
\begin{aligned}
\hat{A} & \equiv \hat{A}(\alpha, \gamma):=\lambda_{1}+\frac{\delta}{1-\delta} \eta_{0} \sigma_{0}^{\top}\left(\sigma_{0} \sigma_{0}^{\top}\right)^{-1} \theta_{1}, \\
\hat{R} & \equiv \hat{R}(\alpha, \gamma):=\left(I+\frac{\delta}{1-\delta} \sigma_{0}^{\top}\left(\sigma_{0} \sigma_{0}^{\top}\right)^{-1} \sigma_{0}\right)^{-1}, \\
\hat{C} & \equiv \hat{C}(\alpha, \gamma):=\gamma r_{2}+\frac{\delta}{1-\delta} \theta_{1}^{\top}\left(\sigma_{0} \sigma_{0}^{\top}\right)^{-1} \theta_{1}, \\
\hat{d} & \equiv \hat{d}(\alpha, \gamma):=\lambda_{0}+\frac{\delta}{1-\delta} \eta_{0} \sigma_{0}^{\top}\left(\sigma_{0} \sigma_{0}^{\top}\right)^{-1} \theta_{0}, \\
\hat{e} & \equiv \hat{e}(\alpha, \gamma):=\gamma r_{1}+\frac{\delta}{1-\delta} \theta_{1}^{\top}\left(\sigma_{0} \sigma_{0}^{\top}\right)^{-1} \theta_{0}, \\
\hat{f} & \equiv \hat{f}(\alpha, \gamma):=\gamma r_{0}+\frac{\delta}{1-\delta} \theta_{0}^{\top}\left(\sigma_{0} \sigma_{0}^{\top}\right)^{-1} \theta_{0} .
\end{aligned}
$$

Here $I$ denotes the identity matrix of appropriate dimension. The ergodic-type Bellman equation associated with the infinite time horizon problem (1.8) is now deduced as the limit equation of (2.8) as $T-t \rightarrow \infty$, by assuming that $v(t, y) \sim \chi(T-t)+\xi(y), \partial_{t} v(t, y) \rightarrow-\chi$, $\partial_{y} v(t, y) \rightarrow \nabla \xi(y)$, and $\partial_{y y} v(t, y) \rightarrow \nabla \nabla \xi(y)$, as $T-t \rightarrow \infty$, where $\chi$ is some constant and $\xi(\cdot)$ is twice differentiable. We deduce that

$$
\chi=\frac{1}{2} \operatorname{Tr}\left(\eta_{0} \eta_{0}^{\top} \nabla \nabla \xi\right)+\frac{1}{2}(\nabla \xi)^{\top} \eta_{0} \hat{R}^{-1} \eta_{0}^{\top} \nabla \xi+a(y)^{\top} \nabla \xi+b(y)
$$


or, equivalently, that

$$
\chi=\frac{1}{2}\left\{\operatorname{Tr}\left(\eta_{0} \eta_{0}^{\top} \nabla \nabla \xi\right)+\left|\eta_{0}^{\top} \nabla \xi\right|^{2}\right\}+\max _{\pi \in \mathbb{R}^{n}}\left\{\left(\lambda(y)+\delta \eta_{0} \sigma_{0}^{\top} \pi\right)^{\top} \nabla \xi+\delta \ell(y, \pi)\right\},
$$

recalling that the maximizer is

$$
\check{\pi}(t, y):=\frac{1}{1-\delta}\left(\sigma_{0} \sigma_{0}^{\top}\right)^{-1}\left\{\theta(y)+\left(\sigma_{0} \eta_{0}^{\top}\right) \nabla \xi(y)\right\} .
$$

Indeed, from a suitable solution, $(\hat{\chi}, \hat{\xi}) \in \mathbb{R} \times C^{2}\left(\mathbb{R}^{m}\right)$, to the ergodic-type Bellman equation (2.10), where $C^{2}(A)$ denotes the space of twice-continuously differentiable functions with (generic) domain $A$, we can construct a solution to problem (1.8). That is, letting

$$
\hat{\pi}_{t}:=\bar{\pi}\left(t, Y_{t}\right), \quad \text { where } \bar{\pi}(t, y):=\frac{1}{1-\delta}\left(\sigma_{0} \sigma_{0}^{\top}\right)^{-1}\left\{\theta(y)+\left(\sigma_{0} \eta_{0}^{\top}\right) \nabla \hat{\xi}(y)\right\},
$$

we have

$$
\Lambda_{\alpha}(\gamma)=\hat{\chi}<\infty \text { and } \hat{\pi} \in \mathcal{A} \text { given by (2.12) is optimal for (1.8). }
$$

Before stating our result, we recall some notions about matrix-valued algebraic Riccati equations of the form

$$
A^{\top} Q+Q A+Q B R^{-1} B^{\top} Q+C=0
$$

where $A, C \in \mathbb{R}^{m \times m}, B \in \mathbb{R}^{m \times l}, R \in \mathbb{R}^{l \times l}$, and $C$ and $R$ are symmetric. A real, symmetric solution, $Q$, to (2.14) is called stabilizing if $A+B R^{-1} B^{\top} Q$ is stable. It can be shown that the stabilizing solution is uniquely determined if it exists. (See Theorem 5 of [20] or Theorem 23.4 of [3]. For an extensive treatment of algebraic Riccati equations, we refer the reader to [15], for example.) We denote the stabilizing solution by

$$
\operatorname{Ric}\left(\begin{array}{cc}
A & B R^{-1} B^{\top} \\
-C & -A^{\top}
\end{array}\right)
$$

following convention, since the solution has been well studied through analysis of the associated Hamiltonian matrix,

$$
\left(\begin{array}{cc}
A & B R^{-1} B^{\top} \\
-C & -A^{\top}
\end{array}\right)
$$

Let

$$
\hat{H} \equiv \hat{H}(\alpha, \gamma):=\left(\begin{array}{cc}
\hat{A} & \eta_{0} \hat{R}^{-1} \eta_{0}^{\top} \\
-\hat{C} & -\hat{A}^{\top}
\end{array}\right),
$$

where the notation is as defined in (2.9). We obtain the following theorem.

Theorem 2.1. Assume that (2.1)-(2.3) hold and recall the definitions in (2.6) and (2.9). Let $(\alpha, \gamma) \in[0,1) \times(0,1 /(1-\alpha))$ be given. Suppose that there exists a $\hat{Q}:=\operatorname{Ric} \hat{H}>0$. Let $\hat{q} \in \mathbb{R}^{m}$ satisfy

$$
\left(\hat{A}+\eta_{0} \hat{R}^{-1} \eta_{0}^{\top} \hat{Q}\right)^{\top} \hat{q}+\hat{Q} \hat{d}+\hat{e}=0
$$

and set

$$
\begin{aligned}
\hat{\chi} & :=\frac{1}{2} \operatorname{Tr}\left(\eta_{0} \eta_{0}^{\top} \hat{Q}\right)+\frac{1}{2} \hat{q}^{\top} \eta_{0} \hat{R}^{-1} \eta_{0}^{\top} \hat{q}+\hat{d}^{\top} \hat{q}+\hat{f}, \\
\hat{\xi}(y) & :=\hat{q}^{\top} y+\frac{1}{2} y^{\top} \hat{Q} y .
\end{aligned}
$$

The pair $(\hat{\chi}, \hat{\xi}) \in \mathbb{R} \times C^{2}\left(\mathbb{R}^{m}\right)$ solves (2.10) and can be used to construct a solution to problem (1.8), i.e. (2.13) holds. Furthermore, the optimal strategy, $\hat{\pi}$, satisfies (1.11). 
Remark 2.1. The results for Black-Scholes-type models with constant coefficients can be recovered. Assuming that (2.1)-(2.3) hold, let $r_{1}=0, r_{2}=\lambda_{1}=0$, and $\theta_{1}=0$ (so $\hat{A}=\hat{C}=0$ and $\hat{e}=0)$. Trivially, $(\hat{\chi}, \hat{\xi}):=(\hat{f}, 0)$ solves $(2.10)$ and (2.13) follows.

The following necessary and sufficient condition to ensure the existence of

$$
\operatorname{Ric}\left(\begin{array}{cc}
A & B R^{-1} B^{\top} \\
-C & -A^{\top}
\end{array}\right)
$$

is useful for our study.

Theorem 2.2. (Theorem 5 of [20].) Assume that the pair $(A, B) \in \mathbb{R}^{m \times m} \times \mathbb{R}^{m \times l}$ is controllable, i.e. that $\operatorname{rank}\left[B, A B, \ldots, A^{m-1} B\right]=m$. Define

$$
\begin{aligned}
& F(s):=R-B^{\top}\left(-\mathrm{i} s I-A^{\top}\right)^{-1} C(\mathrm{i} s I-A)^{-1} B, \\
& G(s):=B^{\top}\left(-\mathrm{i} s I-A^{\top}\right)^{-1}(\mathrm{i} s I-A)^{-1} B,
\end{aligned}
$$

for $s \in \mathbb{R}$. Then

$$
\operatorname{Ric}\left(\begin{array}{cc}
A & B R^{-1} B^{\top} \\
-C & -A^{\top}
\end{array}\right)
$$

exists if and only if $F(s) \geq \varepsilon G(s)$ for all $s \in \mathbb{R}$ and some $\varepsilon>0$.

With the help of the condition in Theorem 2.2, rewritten to apply in our setting, we obtain the following result.

Theorem 2.3. Assume that (2.1)-(2.3) hold.

1. Let

$$
\tilde{R} \equiv \tilde{R}(\alpha, \gamma):=\left(\eta_{0} \eta_{0}^{\top}\right)^{-1} \eta_{0} \hat{R} \eta_{0}^{\top}\left(\eta_{0} \eta_{0}^{\top}\right)^{-1}
$$

and define, for $s \in \mathbb{R}$,

$$
\begin{aligned}
\hat{N}(s) \equiv \hat{N}(s ; \alpha, \gamma) & :=\left(\begin{array}{cc}
s^{2} \tilde{R}+\left(\hat{A}^{\top} \tilde{R} \hat{A}-\hat{C}\right) & s\left(\hat{A}^{\top} \tilde{R}-\tilde{R} \hat{A}\right) \\
-s\left(\hat{A}^{\top} \tilde{R}-\tilde{R} \hat{A}\right) & s^{2} \tilde{R}+\left(\hat{A}^{\top} \tilde{R} \hat{A}-\hat{C}\right)
\end{array}\right) \\
& =\left(\begin{array}{cc}
s I & \hat{A}^{\top} \\
-\hat{A}^{\top} & s I
\end{array}\right)\left(\begin{array}{cc}
\tilde{R} & 0 \\
0 & \tilde{R}
\end{array}\right)\left(\begin{array}{cc}
s I & -\hat{A} \\
\hat{A} & s I
\end{array}\right)-\left(\begin{array}{cc}
\hat{C} & 0 \\
0 & \hat{C}
\end{array}\right) .
\end{aligned}
$$

Then Ric $\hat{H}$ exists if $\hat{N}(s) \geq \varepsilon I$ for all $s \in \mathbb{R}$ and some $\varepsilon>0$, and is positive definite if $\hat{A}$ is stable.

2. For any given $\alpha_{0} \in[0,1)$, there exist an interval $J \ni \alpha_{0}(J:=(\underline{\alpha}, \bar{\alpha})$ or $J:=[0, \bar{\alpha})$, $0 \leq \underline{\alpha}<\bar{\alpha}<1)$ and $a \bar{\gamma}>0$ such that Ric $\hat{H}(\alpha, \gamma)>0$ exists for any $(\alpha, \gamma) \in J \times[0, \bar{\gamma})$.

We can summarize Theorems 1.1, 2.1, and 2.3.2 and Remark 1.1 as follows.

Corollary 2.1. Assume that (2.1)-(2.3) hold. Let $\alpha \in[0,1)$ be given. Then there exists a $\bar{\gamma}>0$ such that problems (1.7) and (1.8) can be solved for any $\gamma \in(0, \bar{\gamma})$. In particular, with (2.9) and (2.16), $\Gamma_{\alpha}(\gamma)=\Lambda_{\alpha}(\gamma)=\hat{\chi}$ and the strategy $\hat{\pi} \in \mathcal{A}$ is optimal for both (1.7) and (1.8), with respective limits (1.10) and (1.11). Furthermore, problem (1.12) can be solved for any target growth rate $k<\Lambda_{\alpha}^{\prime}(\bar{\gamma}-)$. The 'duality relation' (1.13) holds and a nearly optimal strategy can be constructed using (1.14). 
Remark 2.2. Assertions essentially equivalent to those of Theorem 2.1 and Theorem 2.3.2 were obtained in Theorem 4.6 of [7] (see [6] also), with a different proof, though they treat the $\alpha=0$ case and there are some minor differences in the models. The authors of [7] derived the assertions through a detailed study of the properties of the solution to the ergodic Bellman equation (2.10) (or (2.11)) using a constrained set of trading strategies, $\left\{\pi \in \mathcal{A}:\left|\pi_{t}\right| \leq c\right.$ for all $\left.t \geq 0\right\}$, and relaxing the constraint by letting $c \uparrow \infty$. On the other hand, we directly obtained Theorem 2.3 from linear algebraic arguments (see the proof of Theorem 2.3 (in Section 3), which depends on Theorem 2.2 (i.e. Theorem 5 of [20])). For other related calculations (with $\alpha=0$ ), we refer the reader to [11] and [14]. The latter employs a model similar to ours and succeeds in providing interesting $\gamma$-independent conditions to ensure the solvability of (1.15) for $\gamma<0$, though they are not applicable for $\gamma>0$, which is our case.

Theorem 2.1 implies that showing the existence of $\hat{Q}:=\operatorname{Ric} \hat{H}>0$ is sufficient for constructing the optimal portfolio in (1.8). Combining this with Theorem 2.3, we observe that $\tilde{D}_{\alpha} \subset \mathscr{D}_{\alpha}$, where

$$
\begin{aligned}
& \mathcal{D}_{\alpha}:=\left\{\gamma \in\left(0, \frac{1}{1-\alpha}\right): \Lambda_{\alpha}(\gamma)<\infty\right\}, \\
& \tilde{D}_{\alpha}:=\left\{\gamma \in\left(0, \frac{1}{1-\alpha}\right): \hat{N}(s ; \alpha, \gamma) \geq \varepsilon I \text { for all } s \in \mathbb{R} \text { and some } \varepsilon>0,\right. \\
& \text { and } \hat{A}(\alpha, \gamma) \text { is stable }\} .
\end{aligned}
$$

On the other hand, it still looks unclear in general that the existence of $\hat{Q}$, which is real, symmetric, stabilizing, and positive, is necessary in solving (1.8). If we consider the $m=1$ case, then the algebraic Riccati equation is nothing but a quadratic equation, allowing us to discuss the necessity of $\hat{Q}$ 's existence. We obtain the following theorem.

Theorem 2.4. Assume that (2.1)-(2.3) hold and that $m=1$. Let $\alpha \in[0,1)$ be given. The set $\tilde{D}_{\alpha}$ can be written as

$$
\tilde{D}_{\alpha}=\left\{\gamma \in\left(0, \frac{1}{1-\alpha}\right): \hat{D}(\alpha, \gamma)>0 \text { and } \hat{A}(\alpha, \gamma)<0\right\},
$$

where

$$
\hat{D} \equiv \hat{D}(\alpha, \gamma):=\left[\hat{A}^{2}-\left(\eta_{0} \hat{R}^{-1} \eta_{0}^{\top}\right) \hat{C}\right](\alpha, \gamma) .
$$

Moreover, we have $\tilde{D}_{\alpha} \subset \mathscr{D}_{\alpha} \subset \mathrm{Cl}\left(\tilde{D}_{\alpha}\right)$, where $\mathrm{Cl}$ denotes the closure of its argument in $(0,1 /(1-\alpha))$.

Remark 2.3. Actually, we can obtain the exact form of the set $\tilde{D}_{\alpha}$ and determine the maximal interval $(0, \bar{\gamma})$ included in it, by analysing the boundary behaviour of $\Lambda_{\alpha}^{\prime}(\bar{\gamma}-)$ for solving $(1.12)$. For this, we refer the reader to [9], [10], [17], [18], and [19], for example.

\section{Proofs}

\subsection{Proof of Theorem 1.1}

The proof is along the lines of that of Theorem 5.1 of [5], with minor generalizations. First, recall that $\Gamma_{\alpha}(\gamma), \Lambda_{\alpha}(\gamma)>-\infty$. Indeed, $0 \in \mathcal{A}$ satisfies

$$
\varlimsup_{T \rightarrow \infty} \frac{1}{T} \log \mathrm{E}\left(X_{T}^{\alpha, 0}\right)^{\gamma}=\varlimsup_{T \rightarrow \infty} \frac{1}{T} \log \mathrm{E}\left(x S_{T}^{0}\right)^{\gamma} \geq 0 .
$$


Next we see, in the $\alpha=0$ case, that the assertion of the theorem is trivial from the expressions (1.3) and (1.4). Now let $\alpha \in(0,1)$. For a given $\alpha_{1} \in(0, \alpha]$ and $\pi:=\left(\pi_{t}\right)_{t \geq 0} \in \mathcal{A}$, define

$$
\Xi_{t}^{\left(\alpha, \alpha_{1} ; \pi\right)}:=\left(\tilde{X}_{t}^{\alpha, \pi}-\alpha_{1} \tilde{M}_{t}^{\alpha, \pi}\right)\left(\tilde{M}_{t}^{\alpha, \pi}\right)^{\alpha_{1} /\left(1-\alpha_{1}\right)}
$$

and let $\Xi_{t}^{\alpha, \pi}:=\Xi_{t}^{(\alpha, \alpha ; \pi)}$. We deduce that

$$
\begin{aligned}
\mathrm{d} \Xi_{t}^{\left(\alpha, \alpha_{1} ; \pi\right)} & =\left(\tilde{M}_{t}^{\alpha, \pi}\right)^{\alpha_{1} /\left(1-\alpha_{1}\right)} \mathrm{d} \tilde{X}_{t}^{\alpha, \pi} \\
& =\Xi_{t}^{\left(\alpha, \alpha_{1} ; \pi\right)} \sum_{i=1}^{n}\left(\frac{\tilde{X}_{t}^{\alpha, \pi}-\alpha \tilde{M}_{t}^{\alpha, \pi}}{\tilde{X}_{t}^{\alpha, \pi}-\alpha_{1} \tilde{M}_{t}^{\alpha, \pi}}\right) \pi_{t}^{i} \frac{\mathrm{d} \tilde{S}_{t}^{i}}{\tilde{S}_{t}^{i}},
\end{aligned}
$$

where we use Itô's formula and the relations $\left(\tilde{X}_{t}^{\alpha, \pi}-\alpha_{1} \tilde{M}_{t}^{\alpha, \pi}\right) \mathrm{d} \tilde{M}_{t}^{\alpha, \pi}=0$ and $\tilde{X}_{t}^{\alpha, \pi}-$ $\alpha_{1} \tilde{M}_{t}^{\alpha, \pi}>0$. Rewrite (3.1) as

$$
\Xi_{t}^{\left(\alpha, \alpha_{1} ; \pi\right)}=\left(\tilde{X}_{t}^{\alpha, \pi}\right)^{1 /\left(1-\alpha_{1}\right)} f_{\alpha_{1}}\left(\frac{\alpha_{1} \tilde{M}_{t}^{\alpha, \pi}}{\tilde{X}_{t}^{\alpha, \pi}}\right),
$$

where we define, for $\alpha \in(0,1)$,

$$
f_{\alpha}(x):=(1-x)\left(\frac{x}{\alpha}\right)^{\alpha /(1-\alpha)},
$$

which is decreasing on $(\alpha, \infty)$. Here note that

$$
\alpha_{1} \leq \frac{\alpha_{1} \tilde{M}_{t}^{\alpha, \pi}}{\tilde{X}_{t}^{\alpha, \pi}} \leq \frac{\alpha_{1}}{\alpha} \quad \text { a.e. }(t, \omega)
$$

Letting $\alpha_{1}=\alpha$, we now see, from (3.1)-(3.4), that

$$
(1-\alpha) x^{1 /(1-\alpha)} \mathrm{e}^{L_{t}^{\pi}}=\Xi_{t}^{\alpha, \pi} \leq\left(\tilde{X}_{t}^{\alpha, \pi}\right)^{1 /(1-\alpha)} f_{\alpha}(\alpha),
$$

where $L_{t}^{\pi}$ is as defined in (1.4). We thus deduce that

$$
(1-\alpha)^{(1-\alpha) \gamma} x^{\gamma}\left(S_{T}^{0} \mathrm{e}^{\left.(1-\alpha) L_{T}^{\pi}\right)^{\gamma}} \leq\left(X_{T}^{\alpha, \pi}\right)^{\gamma}\left\{f_{\alpha}(\alpha)\right\}^{\alpha},\right.
$$

which implies that $\Lambda_{\alpha}(\gamma) \leq \Gamma_{\alpha}(\gamma)$. In particular, if $\Lambda_{\alpha}(\gamma)=\infty$ then $\Lambda_{\alpha}(\gamma)=\Gamma_{\alpha}(\gamma)=\infty$.

We next show the reverse inequality, assuming that $\Lambda_{\alpha}(\gamma)<\infty$ and that $\Lambda_{(\cdot)}(\gamma)$ is upper semicontinuous on the left at $\alpha$, as follows. Let $\alpha_{1} \in(0, \alpha)$ be sufficiently close to $\alpha$. From (3.3) and (3.4), we see that

$$
\left(\Xi_{T}^{\left(\alpha, \alpha_{1} ; \pi\right)}\right)^{\left(1-\alpha_{1}\right) \gamma}\left(S_{T}^{0}\right)^{\gamma} \geq\left(X_{T}^{\alpha, \pi}\right)^{\gamma}\left\{f_{\alpha_{1}}\left(\frac{\alpha_{1}}{\alpha}\right)\right\}^{\left(1-\alpha_{1}\right) \gamma} .
$$

Here we note that $f_{\alpha_{1}}\left(\alpha_{1} / \alpha\right)>0$. Write

$$
\rho_{t}^{i} \equiv \rho_{t}^{i}\left(\alpha, \alpha_{1} ; \pi\right):=\left(\frac{\tilde{X}_{t}^{\alpha, \pi}-\alpha \tilde{M}_{t}^{\alpha, \pi}}{\tilde{X}_{t}^{\alpha, \pi}-\alpha_{1} \tilde{M}_{t}^{\alpha, \pi}}\right) \pi_{t}^{i}
$$


for $i=1, \ldots, n$ and $\rho_{t}:=\left(\rho_{t}^{1}, \ldots, \rho_{t}^{n}\right)^{\top}$. Note that

$$
\sum_{i, j=1}^{n} \int_{0}^{T} \rho_{t}^{i} \rho_{t}^{j} \frac{\mathrm{d}\left\langle S^{i}, S^{j}\right\rangle_{t}}{S_{t}^{i} S_{t}^{j}} \leq \sum_{i, j=1}^{n} \int_{0}^{T} \pi_{t}^{i} \pi_{t}^{j} \frac{\mathrm{d}\left\langle S^{i}, S^{j}\right\rangle_{t}}{S_{t}^{i} S_{t}^{j}}<\infty \quad \text { almost surely }
$$

and that

$$
\sum_{i=1}^{n} \int_{0}^{T}\left|\rho_{t}^{i}\right| \frac{\mathrm{d} S_{t}^{0}}{S_{t}^{0}} \leq \sum_{i=1}^{n} \int_{0}^{T}\left|\pi_{t}^{i}\right| \frac{\mathrm{d} S_{t}^{0}}{S_{t}^{0}}<\infty \quad \text { almost surely; }
$$

thus, $\rho \in \mathcal{A}$. We deduce that

$$
\begin{aligned}
\sup _{\pi \in \mathcal{A}} \varlimsup_{T \rightarrow \infty} \frac{1}{T} \log \mathrm{E}\left(\left(\Xi_{T}^{\left(\alpha, \alpha_{1} ; \pi\right)}\right)^{\left(1-\alpha_{1}\right) \gamma}\left(S_{T}^{0}\right)^{\gamma}\right) & =\sup _{\pi \in \mathcal{A}} \varlimsup_{T \rightarrow \infty} \frac{1}{T} \log \mathrm{E}\left(S_{T}^{0} \mathrm{e}^{\left(1-\alpha_{1}\right) L_{T}^{\rho\left(\alpha, \alpha_{1} ; \pi\right)}}\right)^{\gamma} \\
& \leq \Lambda_{\alpha_{1}}(\gamma)
\end{aligned}
$$

where we use (3.1), (3.2), (3.7), and the fact that $\rho\left(\alpha, \alpha_{1} ; \pi\right) \in \mathcal{A}$ for all $\pi \in \mathcal{A}$. Combining (3.6) and (3.8), $\Lambda_{\alpha_{1}}(\gamma) \geq \Gamma_{\alpha}(\gamma)$ follows for any $\alpha_{1} \in(0, \alpha)$. We hence obtain $\Lambda_{\alpha}(\gamma) \geq \overline{\lim }_{\alpha_{1} \uparrow \alpha} \Lambda_{\alpha_{1}}(\gamma) \geq \Gamma_{\alpha}(\gamma)$.

Furthermore, suppose that $\hat{\pi} \in \mathcal{A}$ is optimal for (1.9). We see that

$$
\Gamma_{\alpha}(\gamma)=\Lambda_{\alpha}(\gamma)=\varlimsup_{T \rightarrow \infty} \frac{1}{T} \log \mathrm{E}\left(S_{T}^{0} \mathrm{e}^{\left.(1-\alpha) L_{T}^{\hat{\hat{T}}}\right)^{\gamma}} \leq \varlimsup_{T \rightarrow \infty} \frac{1}{T} \log \mathrm{E}\left(X_{T}^{\alpha, \hat{\pi}}\right)^{\gamma},\right.
$$

where we use (3.5) with $\pi=\hat{\pi}$. Therefore, $\hat{\pi}$ is optimal also for (1.8).

Finally, suppose that $\hat{\pi} \in \mathcal{A}$ satisfies (1.12). We then deduce that

$$
\begin{aligned}
\varlimsup_{T \rightarrow \infty} \frac{1}{T} \log \mathrm{E}\left(X_{T}^{\alpha, \hat{\pi}}\right)^{\gamma} & =\Gamma_{\alpha}(\gamma)=\Lambda_{\alpha}(\gamma)=\underline{\lim } \frac{1}{T \rightarrow \infty} \log \mathrm{E}\left(S_{T}^{0} \mathrm{e}^{(1-\alpha) L_{T}^{\hat{\pi}}}\right)^{\gamma} \\
& \leq \underset{T \rightarrow \infty}{\lim } \frac{1}{T} \log \mathrm{E}\left(X_{T}^{\alpha, \hat{\pi}}\right)^{\gamma}
\end{aligned}
$$

where we use the optimality of $\hat{\pi}$ for (1.8) and the relations (1.10), (1.12), and (3.5) with $\pi=\hat{\pi}$. Therefore, (1.11) follows.

\subsection{Proof of Theorem 2.1}

In this subsection, we assume both that (2.1)-(2.3) always hold and the existence of $\hat{Q}:=$ Ric $\hat{H}(\alpha, \gamma)>0$ for a given $(\alpha, \gamma) \in[0,1) \times(0,1 /(1-\alpha))$, where we use $(2.15)$.

The following result is straightforward.

Lemma 3.1. The pair $(\hat{\chi}, \hat{\xi}) \in \mathbb{R} \times C^{2}\left(\mathbb{R}^{m}\right)$ given by (2.16) solves (2.8).

Proof. The proof follows from direct computations.

Remark 3.1. From the stabilizing property of $\hat{Q}$ and the $\hat{\mathrm{P}}$-ergodicity of (3.12), stated below, the following uniqueness of $(\hat{\chi}, \hat{\xi})$ can be established: if $(\hat{\chi}, \xi) \in \mathbb{R} \times C^{2}\left(\mathbb{R}^{m}\right)$ and $\xi(0)=0$ solves (2.8), then $\xi \equiv \hat{\xi}$. See Theorem 1 of [12].

The proof of Theorem 2.1 can be completed by following that of Theorem 3.1 of [18]. We demonstrate the proof for completeness, combining the following two lemmas.

Lemma 3.2. We have $\Lambda_{\alpha}(\gamma) \leq \hat{\chi}$. 
Proof. Take an arbitrary $\pi \in \mathcal{A}$ and define

$$
G_{t}^{\pi}:=\exp \left\{-\hat{\chi} t+\hat{\xi}\left(Y_{t}\right)-\hat{\xi}\left(Y_{0}\right)+\delta \int_{0}^{t} \ell\left(Y_{u}, \pi_{u}\right) \mathrm{d} u\right\} .
$$

Using Itô's formula, we deduce that

$$
G_{t}^{\pi} \mathcal{E}\left(\delta \int \pi^{\top} \sigma_{0} \mathrm{~d} w\right)_{t}=\mathcal{E}\left(\int\left\{\delta \sigma_{0}^{\top} \pi+\eta_{0}^{\top} \nabla \hat{\xi}(Y)\right\}^{\top} \mathrm{d} w\right)_{t} \exp \left\{-\int_{0}^{t} h_{u} \mathrm{~d} u\right\}
$$

for some nonnegative process $h \geq 0$, since

$$
\begin{aligned}
\frac{\mathrm{d}\left\{G_{t}^{\pi} \mathscr{E}\left(\delta \int \pi^{\top} \sigma_{0} \mathrm{~d} w\right)_{t}\right\}}{G_{t}^{\pi} \mathcal{E}\left(\delta \int \pi^{\top} \sigma_{0} \mathrm{~d} w\right)_{t}}= & \frac{\mathrm{d} G_{t}^{\pi}}{G_{t}^{\pi}}+\frac{\mathrm{d} \mathcal{E}\left(\delta \int \pi^{\top} \sigma_{0} \mathrm{~d} w\right)_{t}}{\mathcal{E}\left(\delta \int \pi^{\top} \sigma_{0} \mathrm{~d} w\right)_{t}}+\frac{\mathrm{d}\left\langle G^{\pi}, \mathcal{E}\left(\delta \int \pi^{\top} \sigma_{0} \mathrm{~d} w\right)\right\rangle_{t}}{G_{t}^{\pi} \mathcal{E}\left(\delta \int \pi^{\top} \sigma_{0} \mathrm{~d} w\right)_{t}} \\
= & \left\{\delta \sigma_{0}^{\top} \pi_{t}+\eta_{0}^{\top} \nabla \hat{\xi}\left(Y_{t}\right)\right\}^{\top} \mathrm{d} w_{t} \\
& +\left[\frac{1}{2}\left\{\operatorname{Tr}\left(\eta_{0} \eta_{0}^{\top} \nabla \nabla \hat{\xi}\left(Y_{t}\right)\right)+\left|\eta_{0}^{\top} \nabla \hat{\xi}\left(Y_{t}\right)\right|^{2}\right\}\right. \\
& \left.+\left(\lambda\left(Y_{t}\right)+\delta \eta_{0} \sigma_{0}^{\top} \pi_{t}\right)^{\top} \nabla \hat{\xi}\left(Y_{t}\right)+\delta \ell\left(Y_{t}, \pi_{t}\right)-\hat{\chi}\right] \mathrm{d} t
\end{aligned}
$$

recalling Lemma 3.1. This implies, from (2.5), that

$$
\begin{aligned}
\log \mathrm{E}\left(S_{T}^{0} \mathrm{e}^{\left.(1-\alpha) L_{T}^{\pi}\right)^{\gamma}}\right. & =\log \mathrm{E} \mathcal{E}\left(\delta \int \pi^{\top} \sigma_{0} \mathrm{~d} w\right)_{T} \exp \left\{\delta \int_{0}^{T} \ell\left(Y_{t}, \pi_{t}\right) \mathrm{d} t\right\} \\
& \leq \hat{\chi} T+\hat{\xi}\left(Y_{0}\right)+\log \mathrm{E} \mathcal{E}\left(\int\left\{\delta \sigma_{0}^{\top} \pi+\eta_{0}^{\top} \nabla \hat{\xi}(Y)\right\}^{\top} \mathrm{d} w\right)_{T} \mathrm{e}^{-\hat{\xi}\left(Y_{T}\right)} .
\end{aligned}
$$

The assertion of the lemma now follows since $\hat{\xi}$ is bounded from below.

Lemma 3.3. We have

$$
\Lambda_{\alpha}(\gamma)=\lim _{T \rightarrow \infty} \frac{1}{T} \log \mathrm{E}\left(S_{T}^{0} \mathrm{e}^{(1-\alpha) L_{T}^{\hat{t}}}\right)^{\gamma}=\hat{\chi}
$$

Proof. Recalling that $\bar{\pi}(y)$ in (2.12) attains the maximum in (2.11) with $\xi=\hat{\xi}$, we deduce that

$$
G_{t}^{\hat{\pi}} \mathcal{E}\left(\delta \int \hat{\pi} \sigma_{0} \mathrm{~d} w\right)_{t}=\mathscr{E}\left(\int\left\{\delta \sigma_{0}^{\top} \hat{\pi}+\eta_{0}^{\top} \nabla \hat{\xi}(Y)\right\}^{\top} \mathrm{d} w\right)_{t},
$$

where we use (3.9) and (3.10). Define the probability measure $\hat{\mathrm{P}}$ on $\left(\Omega, \mathcal{F}_{T}\right)$ by

$$
\left.\frac{\mathrm{d} \hat{\mathrm{P}}}{\mathrm{dP}}\right|_{\mathcal{F}_{t}}:=\mathcal{E}\left(\int\left\{\delta \sigma_{0}^{\top} \hat{\pi}+\eta_{0}^{\top} \nabla \hat{\xi}(Y)\right\}^{\top} \mathrm{d} w\right)_{t}
$$

which is well defined since the integrand in the stochastic exponential on the right-hand side is linear with respect to $Y$. We now see that

$$
\begin{aligned}
\log \mathrm{E}\left(S_{T}^{0} \mathrm{e}^{(1-\alpha) L_{T}^{\hat{r}}}\right)^{\gamma} & =\log \mathrm{E}^{(\delta \hat{\pi})} \exp \left\{\delta \int_{0}^{T} \ell\left(Y_{t}, \hat{\pi}_{t}\right) \mathrm{d} t\right\} \\
& =\hat{\chi} T+\hat{\xi}\left(Y_{0}\right)+\log \hat{\mathrm{E}} \mathrm{e}^{-\hat{\xi}\left(Y_{T}\right)},
\end{aligned}
$$


where we use (2.5) and $\hat{E}$ denotes expectation with respect to $\hat{\mathrm{P}}$. Note here that the dynamics of $Y$ under $\hat{\mathrm{P}}$ is described by

$$
\mathrm{d} Y_{t}=\left\{\left(\hat{d}+\eta_{0} \hat{R}^{-1} \eta_{0}^{\top} \hat{q}\right)+\left(\hat{A}+\eta_{0} \hat{R}^{-1} \eta_{0}^{\top} \hat{Q}\right) Y_{t}\right\} \mathrm{d} t+\eta_{0} \mathrm{~d} \hat{w}_{t},
$$

where, by the Cameron-Martin-Maruyama-Girsanov theorem,

$$
\hat{w}_{t}:=w_{t}-\int_{0}^{t}\left\{\delta \sigma_{0}^{\top} \bar{\pi}\left(Y_{u}\right)+\eta_{0}^{\top} \nabla \hat{\xi}\left(Y_{u}\right)\right\} \mathrm{d} u
$$

is $\hat{\mathrm{P}}$-Brownian motion, recalling that

$$
\begin{aligned}
\lambda(y)+\eta_{0}\left\{\delta \sigma_{0}^{\top} \bar{\pi}(y)+\eta_{0}^{\top} \nabla \hat{\xi}(y)\right\}= & \lambda(y)+\frac{\delta}{1-\delta} \eta_{0} \sigma_{0}^{\top}\left(\sigma_{0} \sigma_{0}^{\top}\right)^{-1} \theta(y) \\
& +\eta_{0}\left\{I+\frac{\delta}{1-\delta} \sigma_{0}^{\top}\left(\sigma_{0} \sigma_{0}^{\top}\right)^{-1} \sigma_{0}\right\} \eta_{0}^{\top} \nabla \hat{\xi}(y) \\
= & \left(\hat{d}+\eta_{0} \hat{R}^{-1} \eta_{0}^{\top} \hat{q}\right)+\left(\hat{A}+\eta_{0} \hat{R}^{-1} \eta_{0}^{\top} \hat{Q}\right) y .
\end{aligned}
$$

Note further that $Y$ is $\hat{\mathrm{P}}$-ergodic, since in (3.12) $\hat{A}+\eta_{0} \hat{R}^{-1} \eta_{0}^{\top} \hat{Q}$ is a stable matrix. Therefore, $\lim _{T \rightarrow \infty}(1 / T) \log \hat{\mathrm{E}} \mathrm{e}^{-\hat{\xi}\left(Y_{T}\right)}=0$, since, from Jensen's inequality and the lower-boundedness of $\hat{\xi}$,

$$
-\frac{1}{T} \hat{\mathrm{E}} \hat{\xi}\left(Y_{T}\right) \leq \frac{1}{T} \log \hat{\mathrm{E}} \mathrm{e}^{-\hat{\xi}\left(Y_{T}\right)} \leq \frac{C_{1}}{T} .
$$

This, together with (3.11), completes the proof.

\subsection{Proof of Theorem 2.3}

Define

$$
\begin{aligned}
& \hat{F}(s) \equiv \hat{F}(s ; \alpha, \gamma):=\hat{R}-\eta_{0}^{\top}\left(-\mathrm{i} s I-\hat{A}^{\top}\right)^{-1} \hat{C}(\mathrm{i} s I-\hat{A})^{-1} \eta_{0}, \\
& \hat{G}(s) \equiv \hat{G}(s ; \alpha, \gamma):=\eta_{0}^{\top}\left(-\mathrm{i} s I-\hat{A}^{\top}\right)^{-1}(\mathrm{i} s I-\hat{A})^{-1} \eta_{0},
\end{aligned}
$$

for $s \in \mathbb{R}$. Furthermore, let

$$
\begin{aligned}
\tilde{F}(s ; \alpha, \gamma): & \left(-\mathrm{i} s I-\hat{A}^{\top}\right)\left(\eta_{0} \eta_{0}^{\top}\right)^{-1} \eta_{0} \hat{F}(s) \eta_{0}^{\top}\left(\eta_{0} \eta_{0}^{\top}\right)^{-1}(\mathrm{i} s I-\hat{A}) \\
& =\left(-\mathrm{i} s I-\hat{A}^{\top}\right)\left(\eta_{0} \eta_{0}^{\top}\right)^{-1} \eta_{0} \hat{R} \eta_{0}^{\top}\left(\eta_{0} \eta_{0}^{\top}\right)^{-1}(\mathrm{i} s I-\hat{A})-\hat{C} \\
& =\left(-\mathrm{i} s I-\hat{A}^{\top}\right) \tilde{R}(\mathrm{i} s I-\hat{A})-\hat{C}
\end{aligned}
$$

where $\tilde{R}$ is as defined in (2.17), and define the quadratic form

$$
\begin{aligned}
U(x, y ; s) \equiv U(x, y ; s, \alpha, \gamma): & =(x-\mathrm{i} y)^{\top} \tilde{F}(s ; \alpha, \gamma)(x+\mathrm{i} y) \\
& =\left(x^{\top}, y^{\top}\right) \hat{N}(s ; \alpha, \gamma)\left(\begin{array}{l}
x \\
y
\end{array}\right)
\end{aligned}
$$

on $\mathbb{C}^{m} \approx \mathbb{R}^{m} \times \mathbb{R}^{m}$, for $(x, y) \in \mathbb{R}^{m} \times \mathbb{R}^{m}$, using (2.18). Recalling that the controllability of $\left(\hat{A}, \eta_{0}\right)$ is always satisfied due to the nondegeneracy of $\eta_{0} \eta_{0}^{\top}>0$, application of Theorem 2.2 shows the existence of $\hat{Q}:=\operatorname{Ric} \hat{H}$. Moreover, we can deduce that $\hat{Q}>0$ in the following way. Note that if $\hat{Q}$ exists then, for each $\tau \in[0,1]$, there exists

$$
\hat{Q}(\tau):=\operatorname{Ric}\left(\begin{array}{cc}
\hat{A} & \eta_{0} \hat{R}^{-1} \eta_{0}^{\top} \\
-\tau \hat{C} & -\hat{A}^{\top}
\end{array}\right) .
$$


Recall that $\hat{Q}(0)=0$ since $\hat{A}$ is stable, and that $\hat{C}>0$. We observe that $(\mathrm{d} / \mathrm{d} \tau) \hat{Q}(\tau)$ satisfies

$$
\left\{\hat{A}+\eta_{0} \hat{R}^{-1} \eta_{0}^{\top} \hat{Q}(\tau)\right\}^{\top} \frac{\mathrm{d}}{\mathrm{d} \tau} \hat{Q}(\tau)+\frac{\mathrm{d}}{\mathrm{d} \tau} \hat{Q}^{\top}(\tau)\left\{\hat{A}+\eta_{0} \hat{R}^{-1} \eta_{0}^{\top} \hat{Q}(\tau)\right\}+\hat{C}=0,
$$

i.e.

$$
\frac{\mathrm{d}}{\mathrm{d} \tau} \hat{Q}(\tau)=\int_{0}^{\infty} \exp \left\{\left(\hat{A}+\eta_{0} \hat{R}^{-1} \eta_{0}^{\top} \hat{Q}(\tau)\right)^{\top} t\right\} \hat{C} \exp \left\{\left(\hat{A}+\eta_{0} \hat{R}^{-1} \eta_{0}^{\top} \hat{Q}(\tau)\right) t\right\} \mathrm{d} t,
$$

which implies that $\hat{Q}(\cdot)$ is monotone increasing in a matrix sense. Therefore, the first assertion is established.

To show the second assertion, let an $\alpha_{0} \in[0,1)$ be given. Recall that $\hat{A}\left(\alpha_{0}, 0\right)=\lambda_{1}$. Choose an interval $\tilde{J} \ni \alpha_{0}\left(\tilde{J}:=\left(\alpha_{1}, \alpha_{2}\right)\right.$ or $\left.\tilde{J}:=\left[0, \alpha_{2}\right), 0 \leq \alpha_{1}<\alpha_{2}<1\right)$ and a $\tilde{\gamma}>0$ such that $\hat{A}(\alpha, \gamma)$ is stable for any $(\alpha, \gamma) \in \tilde{J} \times(0, \tilde{\gamma})$. Denote by $\bar{r} \equiv \bar{r}(\alpha, \gamma)$ the maximal eigenvalue of $\tilde{R}(\alpha, \gamma)>0$. From (2.18), we now observe that

$$
\begin{aligned}
\tilde{U}(x, y ; s, \alpha, \gamma) & :=U(x, y ; s, \alpha, \gamma)+\left(x^{\top}, y^{\top}\right)\left(\begin{array}{cc}
\hat{C}(\alpha, \gamma) & 0 \\
0 & \hat{C}(\alpha, \gamma)
\end{array}\right)\left(\begin{array}{l}
x \\
y
\end{array}\right) \\
& \geq \frac{1}{\bar{r}}\left|s Z_{1}+Z_{2}\right|^{2} \\
& \geq \frac{1}{\bar{r}\left|Z_{1}\right|^{2}}\left\{\left|Z_{1}\right|^{2}\left|Z_{2}\right|^{2}-\left(Z_{1} \mid Z_{2}\right)^{2}\right\} \\
& >0
\end{aligned}
$$

for all nonzero $(x, y) \in \mathbb{R}^{m} \times \mathbb{R}^{m}, s \in \mathbb{R}$, and $(\alpha, \gamma) \in \tilde{J} \times(0, \tilde{\gamma})$. Here we define

$$
Z_{1}:=\left(\begin{array}{l}
x \\
y
\end{array}\right) \quad \text { and } \quad Z_{2} \equiv Z_{2}(\alpha, \gamma):=\left(\begin{array}{c}
\hat{A}(\alpha, \gamma) y \\
-\hat{A}(\alpha, \gamma) x
\end{array}\right)
$$

and use the notation $(\cdot \mid \cdot)$ for the standard inner product on $\mathbb{R}^{2 m}$. The final (strict) inequality above has been deduced as follows: if $k Z_{1}=Z_{2}$ for some $k \in \mathbb{R}$, then $\hat{A}^{2} x=-k^{2} x$ and $\hat{A}^{2} y=-k^{2} y$. This implies that $x=y=0$, since $\hat{A}^{2}$ does not have nonnegative real eigenvalues, from the stability of $\hat{A}(\alpha, \gamma)$ on $\tilde{J} \times(0, \tilde{\gamma})$. Furthermore, note the following. Suppose that sequences

$$
Z_{1, n}:=\left(\begin{array}{c}
x_{n} \\
y_{n}
\end{array}\right) \in \mathbb{R}^{m} \times \mathbb{R}^{m} \quad \text { and } \quad Z_{2, n}:=\left(\begin{array}{c}
\hat{A} y_{n} \\
-\hat{A} x_{n}
\end{array}\right), \quad n \in \mathbb{N},
$$

satisfy $\lim _{n \rightarrow \infty}\left\{\left|Z_{1, n}\right|^{2}\left|Z_{2, n}\right|^{2}-\left(Z_{1, n} \mid Z_{2, n}\right)^{2}\right\}=0$. Then there exists a sequence $\left(k_{n}\right)_{n \in \mathbb{N}}$ such that $\lim _{n \rightarrow \infty}\left|k_{n} Z_{1, n}-Z_{2, n}\right|=0$, whence $\lim _{n \rightarrow \infty}\left(\hat{A}^{2}+k_{n}^{2} I\right) x_{n}=0$ and $\lim _{n \rightarrow \infty}\left(\hat{A}^{2}+\right.$ $\left.k_{n}^{2} I\right) y_{n}=0$ follow. This implies that $\lim _{n \rightarrow \infty} Z_{1, n}=0$, by the reasoning above. Therefore, we can see that

$$
\begin{aligned}
& \inf \left\{\tilde{U}(x, y ; s, \alpha, \gamma):(x, y, s) \in \mathbb{S}^{m} \times \mathbb{S}^{m} \times \mathbb{R}\right\} \\
& \quad \geq \frac{1}{\bar{r}(\alpha, \gamma)} \min \left\{\left|Z_{2}(\alpha, \gamma)\right|^{2}-\left(Z_{1} \mid Z_{2}(\alpha, \gamma)\right):(x, y) \in \mathbb{S}^{m} \times \mathbb{S}^{m}\right\} \\
& \quad=: \underline{\varepsilon}(\alpha, \gamma) \\
& \quad>0
\end{aligned}
$$


for all $(\alpha, \gamma) \in \tilde{J} \times(0, \tilde{\gamma})$, where $\mathbb{S}^{m}:=\left\{x \in \mathbb{R}^{m}:|x|=1\right\}$. Here note that $\underline{\varepsilon}(\alpha, \gamma)$ is continuous with respect to $\alpha$ and $\gamma$.

We finish the proof as follows. Let $\bar{c}(\alpha, \gamma)$ be the maximal eigenvalue of $\hat{C}(\alpha, \gamma) \geq 0$. Recall that $\bar{c}(\alpha, 0)=0$ since $\hat{C}(\alpha, 0)=0$. From (2.18), (3.13), and (3.14), we have $\hat{N}\left(s ; \alpha_{0}, 0\right) \geq$ $(\underline{\varepsilon}(\alpha, \gamma)-\bar{c}(\alpha, \gamma)) I$ for all $s \in \mathbb{R}$ and $(\alpha, \gamma) \in \tilde{J} \times(0, \tilde{\gamma})$. Since $\underline{\varepsilon}(\alpha, 0)-\bar{c}(\alpha, 0)>0$, we can choose an interval $J \times(0, \bar{\gamma}) \subset \tilde{J} \times(0, \tilde{\gamma})$ such that $\underline{\varepsilon}(\alpha, \gamma)-\bar{c}(\alpha, \gamma)>0$ for all $(\alpha, \gamma) \in J \times(0, \bar{\gamma})$. The first assertion of the theorem can then be applied to deduce the second, completing the proof.

\subsection{Proof of Theorem 2.4}

Throughout this subsection we assume that (2.1)-(2.3) hold and that $m=1$, and let a fixed $\alpha \in[0,1)$ be given.

We begin by noting expression (2.19) for the set $\tilde{D}_{\alpha}$. Indeed, for the matrix $\hat{N}(s):=$ $\hat{N}(s ; \alpha, \gamma)$ given by $(2.18)$, we have

$$
\hat{N}(s)=s^{2}\left(\begin{array}{cc}
\tilde{R} & 0 \\
0 & \tilde{R}
\end{array}\right)+\left(\begin{array}{cc}
\hat{A}^{\top} \tilde{R} \hat{A}-\hat{C} & 0 \\
0 & \hat{A}^{\top} \tilde{R} \hat{A}-\hat{C}
\end{array}\right) .
$$

Thus, the condition that $\hat{N}(s) \geq \varepsilon I$ for all $s \in \mathbb{R}$ and some $\varepsilon>0$ is replaced by the condition that $\hat{A}^{\top} \tilde{R} \hat{A}-\hat{C}>0$, which is nothing but the condition that $\hat{D}>0$, where we use $(2.17)$ and (2.20). We also recall, for $\gamma \in \tilde{D}_{\alpha}$, that the stabilizing solution to the algebraic Riccati equation (i.e. the quadratic equation)

$$
\left(\eta_{0} \hat{R}^{-1} \eta_{0}^{\top}\right) Q^{2}+2 \hat{A} Q+\hat{C}=0
$$

is expressed as

$$
\hat{Q}:=\operatorname{Ric} \hat{H}=\left(\eta_{0} \hat{R}^{-1} \eta_{0}^{\top}\right)^{-1}(-\hat{A}-\sqrt{\hat{D}}),
$$

and that it actually satisfies $\hat{Q}>0$ if $\hat{A}<0$.

Now, to prove the theorem, it suffices to show that $\mathscr{D}_{\alpha} \subset \mathrm{Cl}\left(\tilde{D}_{\alpha}\right)$, since we have already observed that $\tilde{D}_{\alpha} \subset \mathscr{D}_{\alpha}$. We prepare three lemmas, as follows.

Lemma 3.4. Assume that $\gamma \in \tilde{D}_{\alpha}$. For any given $T>0$, the function

$$
\hat{v}^{(T)}(t, y):=\hat{\chi}(T-t)+\hat{\xi}(y)+\hat{L}(T-t, y),
$$

where $(\hat{\chi}, \hat{\xi})$ is given by $(2.16)$ and

$$
\begin{aligned}
\hat{L}(t, y) & :=\frac{1}{\hat{k}} \log \hat{\mathrm{E}}\left(\mathrm{e}^{-\hat{k} \hat{\xi}\left(Y_{t}\right)} \mid Y_{0}=y\right), \\
\hat{k} \equiv \hat{k}(\alpha, \gamma) & :=\frac{\eta_{0} \hat{R}^{-1} \eta_{0}^{\top}}{\eta_{0} \eta_{0}^{\top}},
\end{aligned}
$$

solves the Bellman equation (2.7) or, equivalently, (2.8), with the time horizon T.

Proof. Recall that $\hat{L}$ satisfies

$$
\begin{aligned}
\partial_{t} \hat{L} & =\frac{1}{2}\left\{\eta_{0} \eta_{0}^{\top} \partial_{y y} \hat{G}+\eta_{0} \hat{R}^{-1} \eta_{0}^{\top}\left(\partial_{y} \hat{G}\right)^{2}\right\}+\hat{\lambda}(y) \partial_{y} \hat{G}, \quad t \in(0, T], \\
\hat{L}(0, y) & =-\hat{\xi}(y),
\end{aligned}
$$


where

$$
\hat{\lambda}(y):=\left(\hat{d}+\eta_{0} \hat{R}^{-1} \eta_{0}^{\top} \hat{q}\right)+\left(\hat{A}+\eta_{0} \hat{R}^{-1} \eta_{0}^{\top} \hat{Q}\right) y=a(y)+\left(\eta_{0} \hat{R}^{-1} \eta_{0}^{\top}\right) \frac{\mathrm{d}}{\mathrm{d} y} \hat{\xi}(y),
$$

and that the pair $(\hat{\chi}, \hat{\xi})$ solves (2.10) or, equivalently, (2.11), by Lemma 3.1. The current lemma now follows from direct computation.

Now write

$$
\begin{aligned}
\hat{L}(t, y) & =\frac{1}{\hat{k}}\left[-\frac{1}{2} \log \left(1+\hat{k} \hat{Q} \Sigma_{t}^{2}\right)+\frac{\left\{m_{t}(y)-\hat{k} \hat{Q} \Sigma_{t}^{2}\right\}^{2}}{2 \Sigma_{t}^{2}\left(1+\hat{k} \hat{Q} \Sigma_{t}^{2}\right)}-\frac{m_{t}(y)^{2}}{2 \Sigma_{t}^{2}}\right] \\
& =: \hat{g}_{0}(t)+\hat{g}_{1}(t) y+\frac{1}{2} \hat{g}_{2}(t) y^{2},
\end{aligned}
$$

recalling that, under $\hat{\mathrm{P}}, Y_{t}$ is a Gaussian variable with mean $m_{t}(y)$ and variance $\Sigma_{t}^{2}$, where

$$
\begin{aligned}
m_{t}(y) & :=\mathrm{e}^{-\sqrt{\hat{D}} t} y+\frac{\hat{d}+\eta_{0} \hat{R}^{-1} \eta_{0}^{\top} \hat{q}}{\sqrt{\hat{D}}}\left(1-\mathrm{e}^{-\sqrt{\hat{D}} t}\right), \\
\Sigma_{t}^{2} & :=\frac{\eta_{0} \eta_{0}^{\top}}{2 \sqrt{\hat{D}}}\left(1-\mathrm{e}^{-2 \sqrt{\hat{D} t}}\right) .
\end{aligned}
$$

Furthermore, let

$$
\hat{v}^{(T)}(t, y)=\hat{v}_{0}^{(T)}(t)+\hat{v}_{1}^{(T)}(t) y+\frac{1}{2} \hat{v}_{2}^{(T)}(t) y^{2},
$$

where

$$
\begin{aligned}
& \hat{v}_{0}^{(T)}(t):=\hat{\chi}(T-t)+\hat{g}_{0}(T-t), \\
& \hat{v}_{1}^{(T)}(t):=\hat{q}+\hat{g}_{1}(T-t), \\
& \hat{v}_{2}^{(T)}(t):=\hat{Q}+\hat{g}_{2}(T-t) .
\end{aligned}
$$

Note that the triplet $\left(\hat{v}_{2}^{(T)}, \hat{v}_{1}^{(T)}, \hat{v}_{0}^{(T)}\right)$ satisfies the ordinary differential equations

$$
\begin{array}{rlrl}
-\frac{\mathrm{d}}{\mathrm{d} t} v_{2} & =2 \hat{A} v_{2}+\left(\eta_{0} \hat{R}^{-1} \eta_{0}^{\top}\right) v_{2}^{2}+\hat{C}, & & v_{2}(T)=0, \\
-\frac{\mathrm{d}}{\mathrm{d} t} v_{1}=\left(\hat{A}+\eta_{0} \hat{R}^{-1} \eta_{0}^{\top} v_{2}\right) v_{1}+v_{2} \hat{d}+\hat{e}, & v_{1}(T)=0, \\
-\frac{\mathrm{d}}{\mathrm{d} t} v_{0}=\frac{1}{2}\left(\eta_{0} \eta_{0}^{\top}\right) v_{2}+\frac{1}{2}\left(\eta_{0} \hat{R}^{-1} \eta_{0}^{\top}\right) v_{1}^{2}+\hat{d} v_{1}+\hat{f}, & v_{0}(T)=0,
\end{array}
$$

since $\hat{v}^{(T)}$ solves the Bellman equation (2.8).

We now extend the definition of $\hat{v}^{(T)}$ for each given $T>0$. Deducing the expression

$$
\begin{aligned}
\hat{v}_{2}^{(T)}(t) & :=\hat{Q}+\hat{g}_{2}(T-t) \\
& =\hat{Q}+\frac{\mathrm{e}^{-2 \sqrt{\hat{D}}(T-t)}}{\Sigma_{T-t}^{2}\left(1+\hat{k} \hat{Q} \Sigma_{T-t}^{2}\right)}-\frac{\mathrm{e}^{-2 \sqrt{\hat{D}}(T-t)}}{\Sigma_{T-t}^{2}} \\
& =\frac{\hat{C}}{\sqrt{\hat{D}} \operatorname{coth}(\sqrt{\hat{D}}(T-t))-\hat{A}}
\end{aligned}
$$


where we use (2.20) and (3.15)-(3.18), we define

$$
\bar{v}_{2}^{(T)}(t):=\frac{\hat{C}}{\sqrt{\hat{D}} \operatorname{coth}(\sqrt{\hat{D}}(T-t))-\hat{A}}, \quad t \in[0, T),
$$

satisfying $\bar{v}_{2}^{(T)}(T):=\lim _{t \uparrow T} \bar{v}_{2}^{(T)}(t)=0$ as long as $\gamma \in \overline{\mathscr{D}}_{\alpha}^{(T)}$, where

$$
\overline{\mathscr{D}}_{\alpha}^{(T)}:=\left\{\gamma \in\left(0, \frac{1}{1-\alpha}\right): \hat{D}>-\frac{\pi^{2}}{T^{2}} \text { and } \sqrt{\hat{D}} \operatorname{coth}(\sqrt{\hat{D}} T)>\hat{A}\right\} .
$$

We adopt the convention that $0 \operatorname{coth}(0 t)=1 / t=\lim _{x \rightarrow 0} x \operatorname{coth}(x t)$. Furthermore, we define the function $\bar{v}_{1}^{(T)}$ on $[0, T]$ using the second equation in (3.19), letting $v_{2}:=\bar{v}_{2}^{(T)}$, and we define the function $\bar{v}_{0}^{(T)}$ on $[0, T]$ using the third equation in (3.19), letting $v_{i}:=\bar{v}_{i}^{(T)}$ for $i=1,2$. We then have the following result.

Lemma 3.5. Let $T>0$ be given. For $\gamma \in \overline{\mathscr{D}}_{\alpha}^{(T)}$, define

$$
\bar{v}^{(T)}(t, y):=\bar{v}_{0}^{(T)}(t)+\bar{v}_{1}^{(T)}(t) y+\frac{1}{2} \bar{v}_{2}^{(T)}(t) y^{2} .
$$

This solves the Bellman equation (2.7) or, equivalently, (2.8). In addition, $\hat{v}^{(T)} \equiv \bar{v}^{(T)}$ for $\gamma \in \tilde{D}_{\alpha}$.

Using the solution $\bar{v}^{(T)}$ to the Bellman equation (2.7), we can construct the solution to the optimization problem (2.4) with the finite time horizon $T>0$, as follows, via a standard argument in stochastic control theory.

Lemma 3.6. Let $T>0$ and $\gamma \in \overline{\mathscr{D}}_{\alpha}^{(T)}$ be given. Define $\hat{\pi}^{(T)}:=\left(\hat{\pi}_{t}^{(T)}\right)_{t \in[0, T]}$ by

$$
\begin{aligned}
\hat{\pi}_{t}^{(T)} & :=\bar{\pi}^{(T)}\left(t, Y_{t}\right), \\
\bar{\pi}^{(T)}(t, y) & :=\frac{1}{1-\delta}\left(\sigma_{0} \sigma_{0}^{\top}\right)^{-1}\left\{\theta(y)+\sigma_{0} \eta_{0}^{\top} \partial_{y} \bar{v}^{(T)}(t, y)\right\} .
\end{aligned}
$$

For problem (2.4) with $\mathcal{A}_{T}:=\mathcal{L}_{T}^{2}$, the totality of progressively measurable processes on $[0, T]$ which are locally square integrable, we have $\bar{V}_{0}=\bar{v}^{(T)}(0, y)$ and the optimality of $\hat{\pi}^{(T)}$ holds. On the other hand, $\bar{V}_{0}=\infty$ if $\gamma \notin \overline{\mathcal{D}}_{\alpha}^{(T)}$.

Proof. For an arbitrary $\pi \in \mathcal{A}_{T}$, define

$$
H_{t}^{\pi}:=\exp \left\{\bar{v}^{(T)}\left(t, Y_{t}\right)+\delta \int_{0}^{t} \ell\left(Y_{u}, \pi_{u}\right) \mathrm{d} u\right\} .
$$

Using Itô's formula and the fact that $\bar{v}^{(T)}$ solves (2.7), we can check that

$$
H_{t}^{\pi} \mathcal{E}\left(\delta \int \pi^{\top} \sigma_{0} \mathrm{~d} w\right)_{t}=\mathcal{E}\left(\int\left\{\delta \sigma_{0}^{\top} \pi+\eta_{0}^{\top} \partial_{y} \bar{v}^{(T)}\left(\cdot, Y_{(\cdot)}\right)\right\}^{\top} \mathrm{d} w\right)_{t} \exp \left\{-\int_{0}^{t} h_{u} \mathrm{~d} u\right\}
$$

for some nonnegative process $h \geq 0$. This implies that the left-hand side of the above is a supermartingale, and we see that

$$
\mathrm{e}^{\bar{v}^{(T)}\left(0, Y_{0}\right)} \geq \mathrm{E} H_{T}^{\pi} \mathcal{E}\left(\delta \int \pi^{\top} \sigma_{0} \mathrm{~d} w\right)_{T}=\mathrm{E}\left(S_{T}^{0} \mathrm{e}^{(1-\alpha) L_{T}^{\pi}}\right)^{\gamma},
$$


where we use (2.5) and (3.21). Similarly, we can check that

$$
H_{t}^{\hat{\pi}^{(T)}} \mathcal{E}\left(\delta \int\left(\hat{\pi}^{(T)}\right)^{\top} \sigma_{0} \mathrm{~d} w\right)_{t}=\mathcal{E}\left(\int\left\{\delta \sigma_{0}^{\top} \hat{\pi}^{(T)}+\eta_{0}^{\top} \partial_{y} \bar{v}^{(T)}\left(\cdot, Y_{(\cdot)}\right)\right\}^{\top} \mathrm{d} w\right)_{t} .
$$

This implies that the left-hand side of the above is a martingale, since the integrand on the right-hand side has a linear form with respect to $Y$. Therefore,

$$
\begin{aligned}
\mathrm{e}^{\bar{v}^{(T)}\left(t, Y_{t}\right)} & =\mathrm{E}\left(H_{T}^{\hat{\pi}^{(T)}} \mathcal{E}\left(\delta \int\left(\hat{\pi}^{(T)}\right)^{\top} \sigma_{0} \mathrm{~d} w\right)_{T} \mid \mathcal{F}_{t}\right) \\
& =\mathrm{E}\left(\left(S_{t, T}^{0} \exp \left\{(1-\alpha) L_{t, T}^{\hat{\pi}^{(T)}}\right\}\right)^{\gamma} \mid \mathcal{F}_{t}\right)
\end{aligned}
$$

for any $t \in[0, T]$, where we define $S_{t, T}^{0}:=S_{T}^{0} / S_{t}^{0}$ and $L_{t, T}^{\pi}:=L_{T}^{\pi}-L_{t}^{\pi}$. Letting $t=0$, the optimality of $\hat{\pi}^{(T)} \in \mathcal{A}_{T}$ follows.

On the other hand, suppose that $\gamma \notin \bar{D}_{\alpha}^{(T)}$. Then $\bar{v}_{2}^{(T)}\left(t_{0}\right)=\infty$ and $\bar{V}_{t_{0}}=\infty$ for some $t_{0}$, $0<t_{0}<T$, from (3.20) and (3.22), so we deduce that $\bar{V}_{0}=\infty$.

We are now in a position to show the relation $\mathscr{D}_{\alpha} \subset \mathrm{Cl}\left(\tilde{D}_{\alpha}\right)$. Note that

$$
\mathrm{Cl}\left(\tilde{D}_{\alpha}\right)=\left\{\gamma \in\left(0, \frac{1}{1-\alpha}\right): \hat{D}(\alpha, \gamma) \geq 0 \text { and } \hat{A}(\alpha, \gamma) \leq 0\right\}=\bigcap_{T>0} \overline{\mathscr{D}}_{\alpha}^{(T)},
$$

recalling that $\sqrt{\hat{D}} \geq \hat{A}$ if and only if $\hat{A} \leq 0$ with $\hat{D} \geq 0$. Let $\gamma \notin \operatorname{Cl}\left(\tilde{D}_{\alpha}\right)$. From (3.23), there exists a $T_{0}>0$ such that $\gamma \notin \bar{D}_{\alpha}^{(T)}$ for all $T>T_{0}$. This implies that $\gamma \notin \mathscr{D}_{\alpha}$, from Lemma 3.6.

\section{Conclusion}

In the present paper, the maximization of the long-term growth rate of expected utility and the maximization of the large deviation probability of beating the target growth rate in the long run have been treated under drawdown constraints. In a general situation, the values and the optimal strategies of the problems have been related to those of another 'standard' risk-sensitivetype portfolio optimization problem. The risk-aversion parameter of utility was related to the target growth rate, and the drawdown ratio parameter was related to the upper bound of the risk of portfolio loss process. As an example, a model which has a 'linear-quadratic' structure was studied in detail, and explicit expressions of the solutions to the problems presented. In particular, a sufficient condition to ensure the solvability was provided by discussing the existence of the stabilizing solution to a matrix-valued algebraic Riccati equation. A necessary condition for the solvability was also discussed for the one-dimensional Riccati equation.

\section{Acknowledgement}

This study was done while the author was visiting the Department of Mathematics of ETH, Zürich. He wishes to express his thanks for their kind hospitality.

\section{References}

[1] Artzner, P. et al. (2004). Coherent multiperiod risk adjusted values and Bellman's principle. To appear in Ann. Operat. Res.

[2] Bielecki, T. R. ANd Pliska, S. R. (2004). Risk sensitive intertemporal CAPM, with application to fixed-income management. IEEE Trans. Automatic Control 49, 420-432. 
[3] Brockett, R.W. (1970). Finite Dimensional Linear Systems. John Wiley. New York.

[4] Cheridito, P., Delbaen, F. And Kupper, M. (2005). Coherent and convex monetary risk measures for unbounded càdlàg processes. Finance Stoch. 9, 369-387.

[5] Cvitanić, J. And Karatzas, I. (1995). On portfolio optimization under 'drawdown' constraints. In Mathematical Finance (IMA Lecture Notes Math. Appl. 65), Springer, New York, pp. 35-45.

[6] Fleming, W. H. and Sheu, S. J. (2000). Risk-sensitive control and an optimal investment model. Math. Finance 10, 197-213.

[7] Fleming, W. H. And Sheu, S. J. (2002). Risk-sensitive control and an optimal investment model. II. Ann. Appl. Prob. 12, 730-767.

[8] Grossman, S. J. and Zhou, Z. (1993). Optimal investment strategies for controlling drawdowns. Math. Finance 3, 241-276.

[9] Hata, H. AND IIDA, Y. (2005). A risk-sensitive stochastic control approach to an optimal investment problem with partial information. To appear in Finance Stoch.

[10] Hata, H. and Sekine, J. (2005). Solving long term optimal investment problems with Cox-Ingersoll-Ross interest rates. Adv. Math. Econom. 8, 231-255.

[11] Inoue, A. AND NaKano, Y. (2005). Optimal long term investment model with memory. To appear in Appl. Math. Optimization.

[12] KaISE, H. AND Sheu, S. J. (2004). Risk sensitive optimal investment: solutions of the dynamical programming equation. In Mathematics of Finance (Contemp. Math. 351), American Mathematical Society, Providence, RI, pp. 217-230.

[13] Karatzas, I. And Shreve, S. (1991). Brownian Motion and Stochastic Calculus, 2nd edn. Springer, New York.

[14] Kuroda, K. And Nagai, H. (2002). Risk sensitive portfolio optimization on infinite time horizon. Stoch. Stoch. Reports 73, 309-331.

[15] Lancaster, P. and Rodman, L. (1995). Algebraic Riccati Equations. Oxford Science Publications.

[16] NaGaI, H. (2003). Optimal strategies for risk-sensitive portfolio optimization problems for general factor models. SIAM J. Control Optimization 41, 1779-1800.

[17] Pham, H. (2003). A large deviations approach to optimal long term investment. Finance Stoch. 7, 169-195.

[18] Рнам, H. (2003). A risk-sensitive control dual approach to a large deviations control problem. Systems Control Lett. 49, 295-309.

[19] Sekine, J. (2006). On a large deviations control for a linear-quadratic model: the complete dual solution. To appear in Proceedings of the 4th JSIAM-SIMAI Meeting.

[20] Willems, J. C. (1971). Least squares stationary optimal control and the algebraic Riccati equation. IEEE Trans. Automatic Control 16, 621-634. 